\title{
The Study of Morphological Structure, Phase Structure and Molecular Structure of Collagen-PEO 600K Blends for Tissue Engineering Application
}

\author{
${ }^{1}$ N.F. Mohd Nasir, ${ }^{2}$ M.G. Raha, ${ }^{2}$ N.A. Kadri, ${ }^{2}$ S.I. Sahidan, ${ }^{2}$ M.Rampado and ${ }^{3}$ C.A Azlan \\ ${ }^{1}$ Biomedical Electronics Engineering Program, School of Mechatronics \\ Northern Malaysia University College of Engineering, Kangar, Malaysia \\ ${ }^{2}$ Biomedical Engineering Department, Faculty of Engineering \\ University Malaya, Kuala Lumpur, Malaysia \\ ${ }^{3}$ Department of Biomedical Imaging, Faculty of Medicine, University of Malaya \\ Kuala Lumpur, Malaysia
}

\begin{abstract}
A new material which is collagen/ poly (ethylene oxide) (PEO) blend was developed to determine its possibility as a promising material for tissue scaffold. PEO with average molecular weight of 600,000 and collagen originated from calf skin were dispersed in $0.1 \mathrm{M}$ acetic acid to prepare a concentration of $1 \mathrm{wt} \%$ for PEO and $0.15 \mathrm{wt} \%$ for collagen. The collagen-PEO600K blend film was then obtained by solution casting method. SEM results shown that by having certain ratio of collagen and PEO, the membrane began to developed porous structures which are possible to assist tissue attachment on the scaffold. The X-ray diffractograms demonstrate PEO 600K influences on the blend thus enhancing crystallinity of collagen. The Infra red spectrum shows intermolecular interactions of collagen and PEO which alter the collagen structure thus explained the membrane morphological changes. Therefore, we concluded that the phase structure and also the molecular structure of the blend are crucial to produce desirable morphological structure of the membrane which is required for a reliable tissue scaffold.
\end{abstract}

Key words: SEM, XRD, FTIR, PEO, collagen, tissue scaffold

\section{INTRODUCTION}

Collagen is readily available, non-toxic and has the fibril architecture in natural tissues. Thus, collagen provides an excellent basis for biomaterials, such as arterial prostheses and artificial $\operatorname{skin}^{[1,2]}$. The main amino acids in collagen are glycine, proline, hydroxypropline and alanine. The ordered triple helical structure of collagen is stabilized by both interchain hydrogen bonds and by structural water molecules ${ }^{[3-7]}$.

Collagen can be desorbed into the body, is nontoxic, induces only minimal immune response (even between different species) and is excellent for attachment and biological interaction with cells. Collagen may also be processed into a variety of formats, including porous sponges, gels and sheets and can be cross linked with chemicals to make it stronger or to alter its degradation rate ${ }^{[8]}$.

Collagen and PEO do not exist together as blends in nature, but specific properties of each may be used to produce synthetic blends that make unique structure and mechanical properties. The miscibility of PEO and collagen with other synthetic polymers and the properties of the blends have been studied previously ${ }^{[9]}$.
However, specific study which directed to develop a product from this material has not been carried out yet.

Thus, this study is aiming to produce scaffold for tissue engineering application. An ideal scaffold should possess following characteristics for desirable biologic response $^{[5]}$ : (i) three-dimensional and highly porous with an interconnected pore network for cell/tissue growth and flow transport of nutrients and metabolic waste, (ii) biodegradable or bioresorbable with a controllable degradation and resorption rate to match cell/tissue growth in vitro and/or in vivo, (iii) suitable surface chemistry for cell attachment, proliferation and differentiation, (iv) mechanical properties to match those of tissues at the site of implantation and (v) be easily processed to form a variety of shapes and sizes ${ }^{[10]}$.

Therefore, we intend to correlate the membrane surface to their phase structure of collagen/PEO blend membranes which is important in developing a tissue scaffold. This objective is hoped to be achieved through the characterization of the collagen/PEO blend membranes using Scanning Electron Microscopy (SEM), X-Ray Diffraction (XRD) and Fourier Transform Infra Red (FTIR) techniques.

Corresponding Author: N.F. Mohd Nasir, Biomedical Electronics Engineering Program, School of Mechatronic Engineering, Kolej Universiti Kejuruteraan Utara Malaysia (KUKUM), Jalan Kangar-Arau, 02600, Jejawi, Perlis, Malaysia, Tel: +60-4-9798455, Fax: +60-4-9798142 


\section{MATERIAL AND METHODS}

Preparation of collagen, PEO and collagen/PEO blend membranes: Collagen, PEO and collagen/PEO blend membranes were prepared by solution cast method. Collagen (calf skin) from Sigma Aldrich and PEO of molecular weight of 600,000 (Fluka) were dispersed in $0.1 \mathrm{M}$ acetic acid aqueous solution to have a concentration of $0.15 \mathrm{wt} \%$ for collagen and $1 \mathrm{wt} \%$ for PEO. The solutions were continuously stirred with a magnetic stirrer for about 1 hour at room temperature. Blends were prepared by mixing both polymers in 4 different ratios: 1:0, 1:1, 3:1 and 0:1.

\begin{tabular}{lc} 
Table 1: & Ratio of samples \\
\hline Collagen & Poly (ethylene oxide) \\
\hline 1 & 0 \\
1 & 1 \\
3 & 1 \\
0 & 1 \\
\hline
\end{tabular}

Dispersions were cast on a Petri dish and allowed to dry at atmospheric pressure and room temperature to obtain collagen/PEO blend film. Samples were allowed to evaporate slowly at room temperature until a film form on the Petri dish. When the films have dried, the films were peeled according to the size needed for the $\mathrm{XRD}$ and SEM.

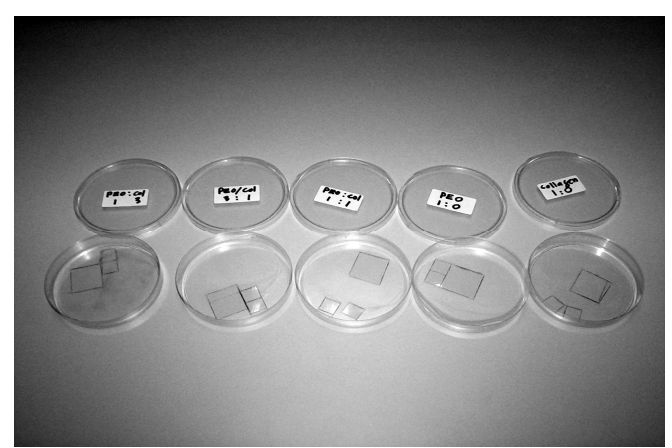

Fig. 1: Collagen/PEO blend samples on Petri dish

Scanning electron microscope (SEM): Morphological properties of the membranes such as surface porosity, roughness and texture were studied. In this study, dried collagen/PEO blend membrane was mounted on a standard SEM sample holder and fixed to the base. The sample was then sputtered coated with gold coating using a SEM coating system before viewed under EDAX scanning electron microscope with accelerating voltage of $10 \mathrm{kV}$.

X-ray diffraction (XRD): Identification and quantitative determination of the various crystalline compounds known as phases were studied using XRD. Thus, the existing phases of developed pure and blend membranes were studied. X-ray diffraction measurements on the membranes were performed by the Siemens XRD Diffractometer.
Fourier transform infra red (FTIR): Characterization of the chemical structure of a polymer is done by using Fourier Transform Infra Red (FTIR) technique. The specific chemistries and orientation of the structure will be known from the IR spectrum. The membranes infra red spectra which are the transmission spectrum were measured using of the Perkin Elmer FTIR Spectrometer.

\section{RESULTS AND DISCUSSION}

Scanning electron microscope (SEM): The morphologies of pure poly (ethylene oxide) $600 \mathrm{~K}$ and pure collagen are featured in the Fig. 2 and 3.

The micrograph shows that PEO $600 \mathrm{~K}$ has crystalline flat lamellae with leave like shape. This feature dominated the morphology of pure PEO membrane. Large spaces between the leave like structure are available through out the surface membrane. The crystalline structure was able to form due to longer evaporation time taken which enabled it to crystallize.

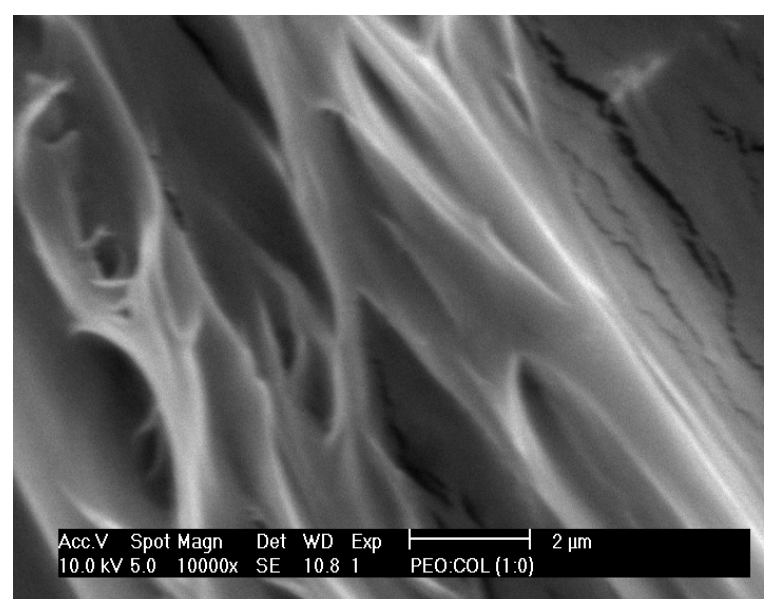

Fig. 2: SEM image of pure PEO 600K membrane

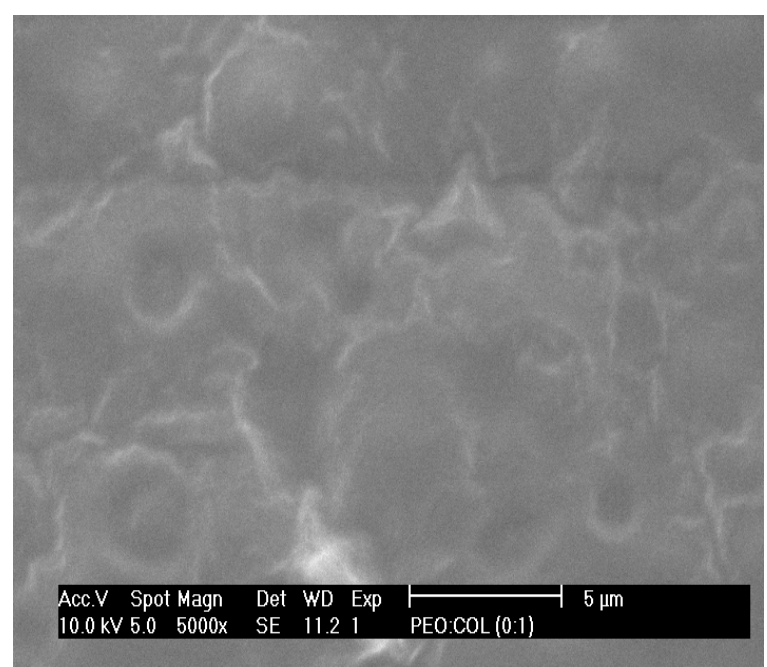

Fig 3: SEM image of pure collagen 


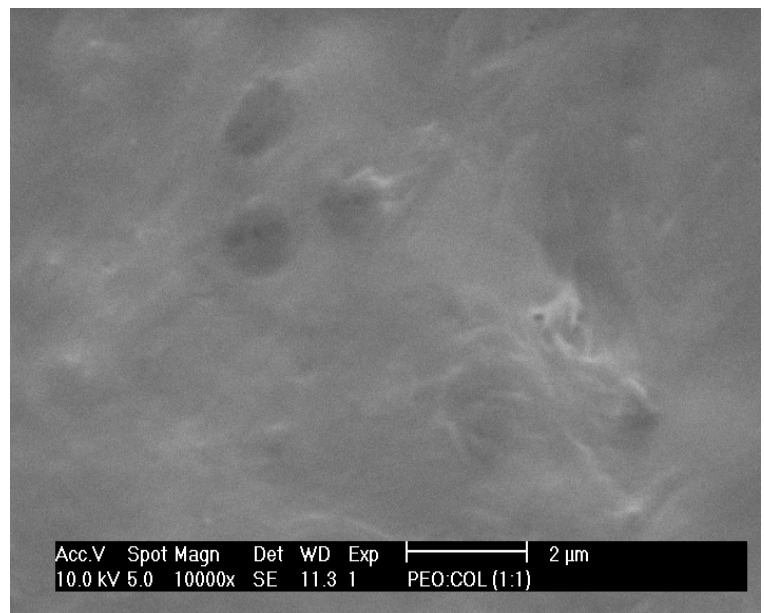

Fig. 4: SEM image of Collagen/PEO 1:1 blend membrane

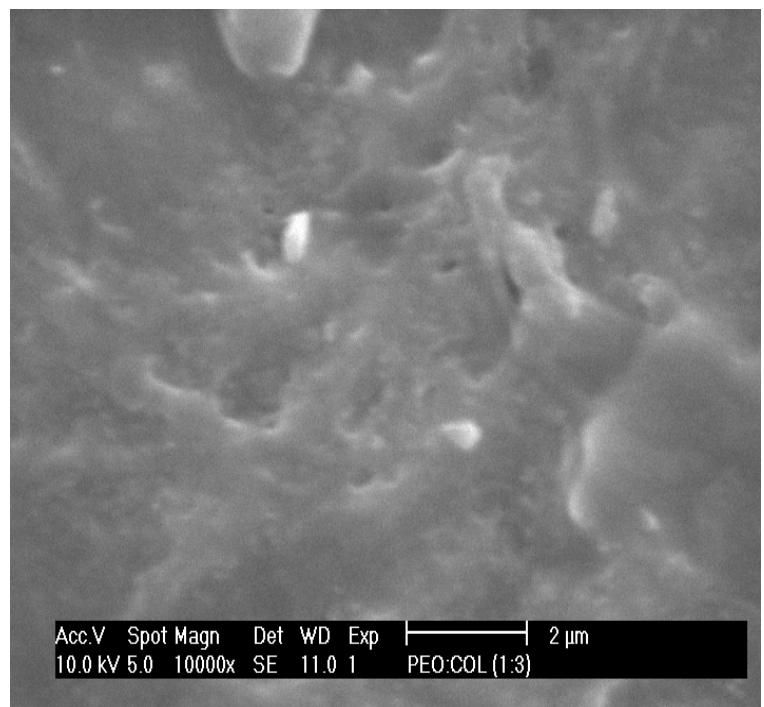

Fig. 5: SEM image of Collagen/PEO 3:1 blend membrane

Meanwhile, for the scanning electron micrograph of pure collagen, it is obvious that the surface is rough and uneven. This feature dominated the surface of pure collagen membrane. However, the membrane is poor in porosity.

For the membrane with collagen/PEO blend ratio 1:1, a much plainer texture could bee seen from Fig. 4. We hope that the crystallinity of PEO could improve the porosity of collagen. However, the image shown had proven that equivalent amount of PEO and collagen in the blend does not contribute to the increment of porosity at the membrane surface.

The micrograph for the collagen/PEO blend membrane with 3:1 ratio shows a better improvement in porosity compared to the previous ratio. Few porous structures began to develop among the uneven structure. The uneven structure is more obvious here compared to the pure collagen surface. Thus, the addition of PEO at the right ratio has improved the

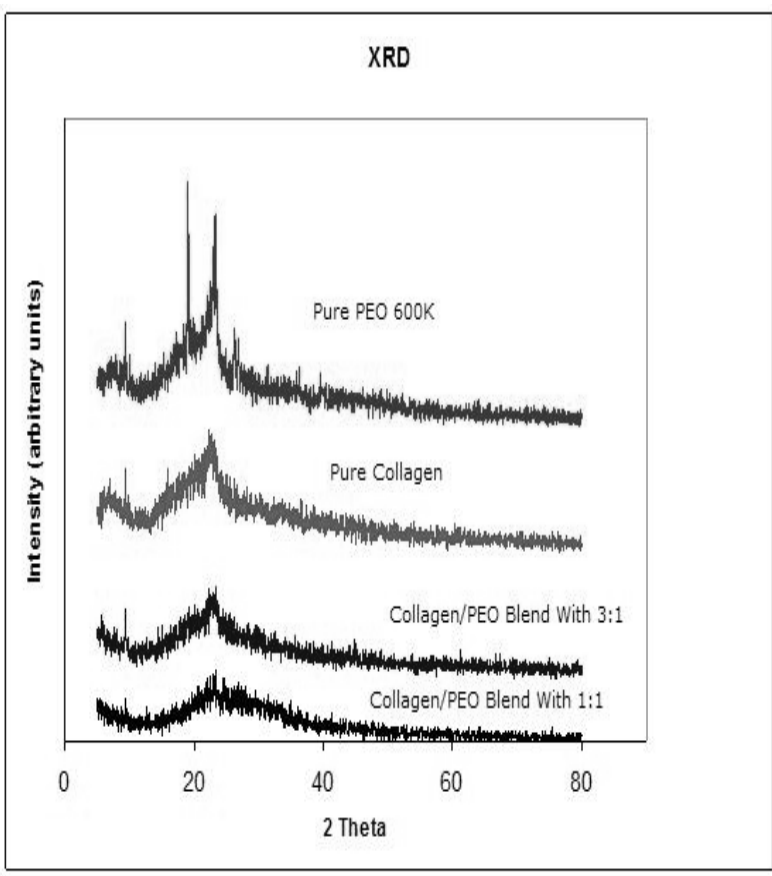

Fig. 6: X-ray diffractograms of pure PEO 600K, pure collagen and collagen/PEO $600 \mathrm{~K}$ blend membranes

morphology of collagen. The porosity of the membrane could be attributed to the membrane's higher degree of crystallinity.

X-Ray Diffraction (XRD): The X-ray diffractograms for collagen, PEO and collagen/PEO blend membranes are shown in Fig. 6. Pure PEO $600 \mathrm{~K}$ membrane exhibits a sharp and intense peak diffractograms at $2 \theta=19^{\circ}$ and $23^{\circ}$ which indicates its high crystallinity. For pure collagen, there is a broad diffraction pattern appeared at $2 \theta=10^{\circ}$ and a characteristic peak which exist at $2 \theta=23^{\circ}$. This broad pattern and characteristic peak indicates a typical diffraction pattern for collagen.

Collagen/PEO blend membrane with 1:1 is still highly amorphous although we expect that equivalent amount of PEO blended with collagen will increase the crystallinity of the membrane.

For the diffractograms of collagen/PEO blend with $3: 1$, the peak at $2 \theta=23^{\circ}$ is more intense and steeper than the peak observed in the previous blend. This indicates that the crystallinity of the membrane has increased.

From the X-ray diffractograms, it is proven that the morphological characteristic of the membrane has direct relationship with the phase structure of the material.

Fourier transform infra red (FTIR): Pure collagen displays bands at 1658,1554 and $1240 \mathrm{~cm}^{-1}$, which are characteristic of the amide I, II and III bands of collagen. The amide I absorption arises predominantly from protein amide $\mathrm{C}=\mathrm{O}$ stretching vibrations, the amide II absorption is made up of amide $\mathrm{N}-\mathrm{H}$ bending 


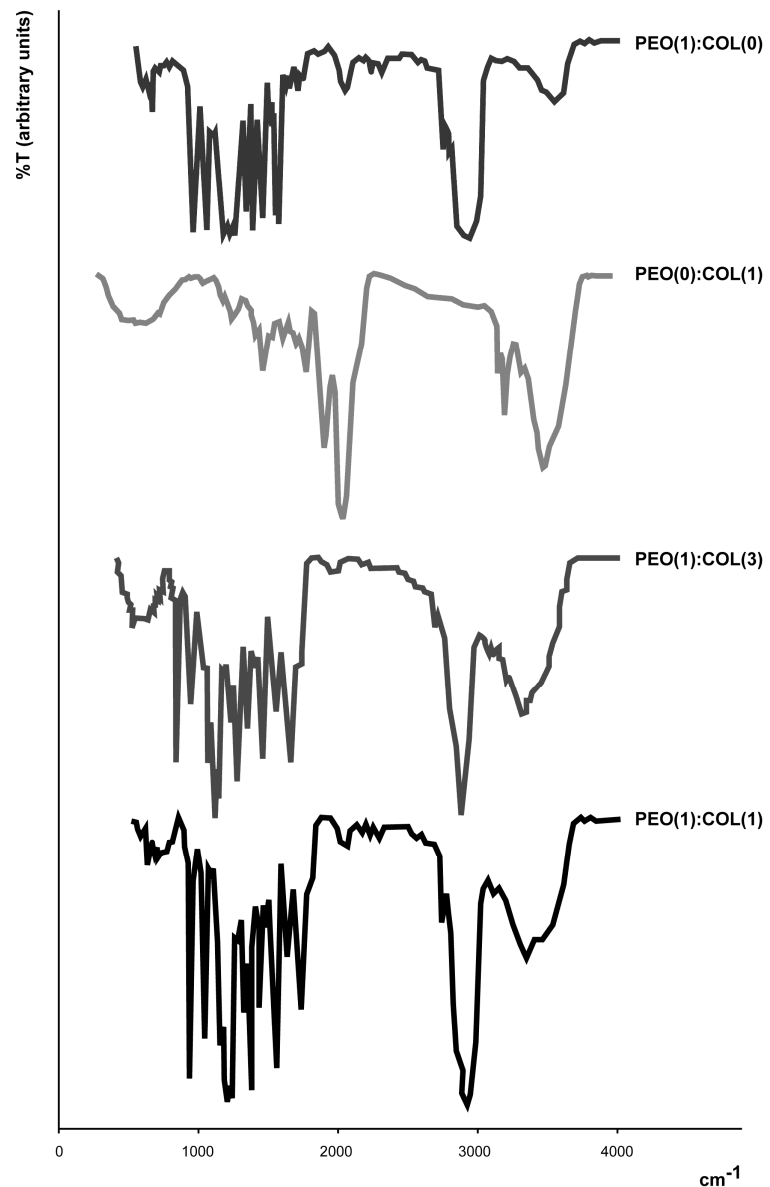

Fig. 7: Infra red spectra of pure PEO 600K, pure collagen and collagen/PEO $600 \mathrm{~K}$ blend membranes

vibrations and $\mathrm{C}-\mathrm{N}$ stretching vibrations (60 and $40 \%$ contribution to the peak, respectively); the amide III peak is complex, consisting of components from $\mathrm{C}-\mathrm{N}$ stretching and $\mathrm{N}-\mathrm{H}$ in plane bending from amide linkages, as well as absorptions arising from wagging vibrations from $\mathrm{CH} 2$ groups from the glycine backbone and proline side-chains ${ }^{[11,12]}$.

The main amide III peak is observed at $1240 \mathrm{~cm}^{-1}$, with smaller peaks seen at 1204 and $1283 \mathrm{~cm}^{-1}$ In collagen the $-\mathrm{OH}$ groups of hydroxyproline are involved in hydrogen bonds between chains, while interactions between other side groups are thought to be important in formation of fibrils from a number of individual molecules ${ }^{[13]}$.

The peak corresponding to the amide I peak displays a change as the level of collagen is reduced relative to the level of PEO in a sample. As the level of collagen in the sample decreases, the amide I peak also decreases.

The amide II peak exhibits intensity relatively with the levels of collagen portion in the blend. The peak corresponding to the amide III peak of collagen display a loss of intensity with increasing content of PEO and decreasing collagen content.
A crystalline PEO phase in the XRD is confirmed by the presence of the triplet peak of the C-O-C stretching vibration at 1149,1109 and $1061 \mathrm{~cm}^{-1}$ with maximum at $1109 \mathrm{~cm}^{-1[14,15]}$. The bands at 947 and 843 $\mathrm{cm}^{-1}$ are known to be characteristic of PEO which influent the crystallinity of the blend. However, the band is much broader in the collagen/PEO 3:1 ratio than the blend with equivalent ratio. This explains the increase of crystallinity of higher amount of collagen in the blend.

\section{CONCLUSION}

Experimental results showed that, by blending certain amount of PEO $600 \mathrm{~K}$ to collagen, the porosity of collagen has improved. The beginning of the formation of pores at the surface of the collagen/PEO 3:1 membrane is resulted from the complexation of two materials. The porosity and the existence of uneven structure at the membrane surface were increased maybe due to the blend membrane's higher crystallinity character. The blending of collagen and PEO at with certain ratio has introduced desirable molecular interactions which facilitate the preliminary formation of pores. Therefore, we concluded that the correlation of the phase structure and chemical structure with the porosity discovered in this research is important to understand the properties of collagen/PEO blend as a promising tissue engineering scaffold. However, as in our previous research ${ }^{[16]}$, we suggest that by blending lower molecular weight PEO with collagen might give a better porosity for the proposed application.

\section{ACKNOWLEDGEMENT}

The authors would like to thank Faculty of Engineering and Faculty of Science of Universiti Malaya for providing facilities and permission to carry out work. The authors are also grateful to the Dean of Mechatronics School, Northern Malaysia University College of Engineering (KUKUM), Prof. Dr. Sazali Yaacob, Dean of Research and Development Unit, Northern Malaysia University College of Engineering (KUKUM), Associate Professor Dr. Uda Hashim and the Head of Department of Biomedical Engineering, Universiti Malaya, Dr Noor Azuan Abu Osman for their continuous support.

\section{REFERENCES}

1. Huc, A., 1985. Collagen biomaterial characteristics and applications. J. Am. Leather Chem. Assoc., 80: 195-212.

2. Matsumoto, K, T. Nakamura, Y. Shimizu, H. Ueda, T. Sekine, Y. Yamamoto, Y. Yiyotami and Y. Takomoto, 1999. A novel surgical material made from collagen with high mechanical strength: A collagen sandwich membrane. OSAIO J. 45: 288292. 
3. Bailey, A.J. and R.G. Paul, 1998. Collagen-is not so simple protein. J. Soc. Leather Technol. Chem., 82: 104-108.

4. Van der Rest, R. and M. Garrone, 1991. Collagen family of proteins. FASEB J., 5: 2814-2823.

5. Ellis, D.O. and S. AcGavin, 1970. The structure of collagen-on X-ray study.J. Ultrastruct. Res., 32: 191-211.

6. Prockop, J. and A. Fertala, 1998. The collagen fibril: The almost crystalline structure. J. Struct. Biol., 122: 111-118.

7. Speer, D.P., M. Chvapil, C.D. Eskelson and J. Vlreich, 1980. Biological effects of residual glutaraldyde in glutaraldehyde-tanned collagen biomaterials. J. Biomed. Mater. Res., 14: 753-764.

8. Cascone, M.G., B. Sim and S. Downes, 1995. Blends of synthetic and natural polymers as drug delivery systems for growth hormone. Biomaterials, 16: 569-574.

9. Sionkowska, A., 2006. Photochemical stability of collagen/PEO blends. J. Photochem. Photobiol. Chem., 177: 61-67.

10. Marovdas, N.G., 1973. Chemical \& mechanical requirements for fibroblast adhesion. Nature, 244: 353.

11. Jackson, M., L.P. Choo, P.H. Watson, W.C. Halliday and H.H. Mantsch, 1995. Beware of connective tissue proteins: assignment and implications of collagen absorptions in infrared spectra of human tissues. Biochim. Biophys. Acta, 1270: 1-6.
12. Renugopalakrishnan, V., G. Chandrakasan, S. Moore, T.B. Hutson, C.V. Berney and R.S. Bhatnagar, 1989. Bound water in collagen. Evidence from Fourier transform infrared and Fourier transform infrared photoacoustic spectroscopic study, Macromolecules, 22: 4121-4.

13. Sionkowska, A., M. Wisniewski, J. Skopinska, C.J. Kennedy and T.J. Wess, 2004. Molecular interactions in collagen and chitosan blends. Biomaterials, 25: 795-801.

14. Tancred, D.C., B.AO. McCormack and A.J. Carr, 1998. A synthetic bone implant macroscopically identical to cancellous bone. Biomaterials, 19: 2303-2311.

15. Yang, S., K.F. Leong, Z. Du and C.K. Chua, 2001. The design of scaffolds for use in tissue engineering. Part I. Traditional factors. Tissue Eng., 7: 679-689.

16. Mohd Nasir, N.F., N. Mohd Zain, M.G. Raha and N.A. Kadri, 2005. Characterization of Chitosanpoly(Ethylene Oxide) blends as haemodialysis membrane. Am. J. Applied Sci., 2: 1578-1583. 